\title{
Design and Development of a Web-based Student Screening Module as Part of a Personalized Learning System
}

\author{
Pavel Zlatarov ${ }^{1}$, Ekaterina Ivanova ${ }^{2}$, Galina Ivanova ${ }^{1}$, Julia Doncheva ${ }^{2}$ \\ ${ }^{I}$ Department of Computing, University of Ruse, 8 Studentska str., Ruse, Bulgaria \\ ${ }^{2}$ Department of Pedagogy, Psychology and History, University of Ruse, 8 Studentska str., Ruse, Bulgaria
}

\begin{abstract}
Various researchers, institutions and companies have been increasingly working on and using e-learning systems in the past. However, with the recent developments, the demand for learning systems that can adapt to learners' need and development level has risen considerably. A lot of learning from a distance requires new approaches in teaching, It is more important than ever for teachers to be able to accurately test students' knowledge, determine the appropriate level of difficulty and adjust content accordingly. This paper describes the design, development and use of a web-based application used to prepare tests for students and determine their level as a module of an integrated personalized learning system. Results from a practical implementation of the system are also discussed.
\end{abstract}

Keywords - personalized learning, e-learning, screening, electronic testing, difficulty level.

\section{Introduction and Background}

Over the past couple of decades, interest in webbased learning and innovative educational tools has spiked tremendously, especially during the recent pandemic conditions [1], [2], [3], [4].

DOI: 10.18421/TEM103-58

https://doi.org/10.18421/TEM103-58

Corresponding author: Pavel Zlatarov, Department of Computing, Faculty of Electrical Engineering, Electronics and Automation, University of Ruse, Bulgaria.

Email: pzlatarov@uni-ruse.bg

Received: 09 June 2021.

Revised: 14 August 2021.

Accepted: 19 August 2021.

Published: 27 August 2021.

(c) BY-NC-ND (c) 2021 Pavel Zlatarov et al; published by UIKTEN. This work is licensed under the Creative Commons Attribution-NonCommercial-NoDerivs 4.0 License.

The article is published with Open Access at www.temjournal.com
Virtual learning environments and interactive tools have the potential to help educators break free from the conventional approach, where curriculum and learning content is often prepared to be the same for every student, not considering each student's development level and individual needs [5] .

The non-personalized approach has been found to, in certain cases, cause poor retention, less accurate testing, and, sometimes, even information overload [6]. These shortcomings have led researchers and educators into studying and developing novel, personalized learning methods, designed to adapt different aspects of the educational process to the needs of the individual learner; this may involve finding suitable curriculum sequences (personalized learning paths, including content and exercise, matching the individuals' needs closely enough) [7], utilizing suitable presentation techniques, making use of software with adaptive user interface elements and more.

With rapid advances in technology, educators have increasingly been shifting to E-learning systems; these systems have been gaining a lot of momentum over the years, but traditional examples primarily fall into the Learning Management System category, where content and curriculum are presented in the same way for each learner, regardless of their interests, development level, particular skills or other individual characteristics [8]. Personalized learning systems attempt to solve this problem by evaluating the learner's individual needs and adapting curriculum, content, elements of the user interface and other aspects of the system to better suit the learner [7],[8].

A screening system can be an integral part of personalized learning in the sense that it can be used in situations where there is a requirement to accurately determine the students' development level and relevant skills, thus enabling related systems to provide them with a personalized learning path, relevant content, and tasks.

In addition, the use of computer technology helps students to upgrade and develop their creative 
thinking in a slightly more non-standard way, but at the same time helps them to express their individuality according to their interests and needs [9].

Such solutions may process existing data that may have been collected, such as tests that have been conducted with learners, or directly aid in conducting the screening process. Information systems have proved valuable in data collection and processing in various situations, from education to medicine. [10], [11]. One such system, in the context of education of primary school students, is presented in this paper.

\section{Proposed Solution}

The developed screening system is part of a personalized learning system (PLS). The PLS has initially been developed to serve students with special needs, but has since been expanded to support elementary and secondary school students. Similar systems have also been developed to serve higher education institutions, notably doctoral students [12].

Personalized learning systems need to adapt to a learner's individual needs; thus, they need to store individual profiles. However, it is not only learners that need a profile - each teacher, lecturer, tutor, specialist, etc. involved in the education process needs their own profile. A learner's profile may include the following basic information:

- Name

- Age

- Grade and school

- Gender

- Authentication credentials such as email, username, password

- Teachers' notes

The user profile creation screen is shown in Figure 1. It allows administrative users, including teachers, to create new profiles for students and/or other user types, such as parents, if applicable.

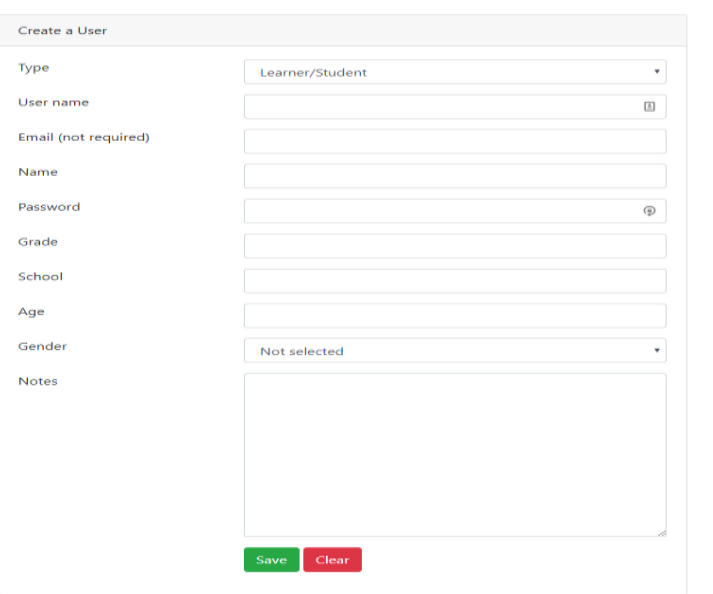

Figure 1. The user profile creation screen
Since user profiles may include certain kinds of personal information, caution should be used wherever there exist legislative frameworks regarding data protection [13]. In such cases, schools and organizations using the personalized learning system should be registered as personal information administrators, and preferably anonymize as much of the personally identifiable information as possible.

In addition to basic information, learners' profiles contain data from different modules. Each module, while relatively independent, can have a varying degree of access to a profile. For example, the screening system can push individual test results to the user profile. Access to profiles is strictly controlled and each module can only request access to information and features it needs. Both the core of the PLS and the modules implement the OAuth2 protocol to request and manage access. Administrators can add new modules and define levels of access, while users can allow or deny access to their profile by each module.

The screening system supports the creation of userdefined tests that may be filled out by students - both electronically and on paper. Teachers may create as many tests and add as many questions as they need. The test creation screen is shown in Figure 2.

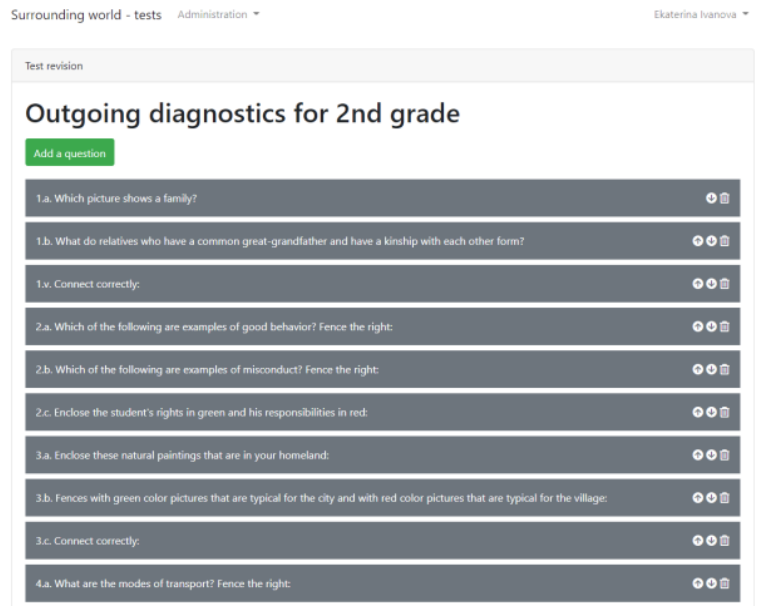

Figure 2. The test creation screen

To make test creation user-friendly, the test creation process supports:

- Adding new questions. Questions can be any of the following types:

- Single-choice - students can only choose one correct answer;

○ Multiple-choice - students may select one or more correct answer;

- Connect pairs - students can pair options to one another to indicate answers;

- Group - students may group answers into different categories.

- Re-ordering questions.

- Editing and deleting questions. 
The question creation/edit screen is shown in Figure 3.

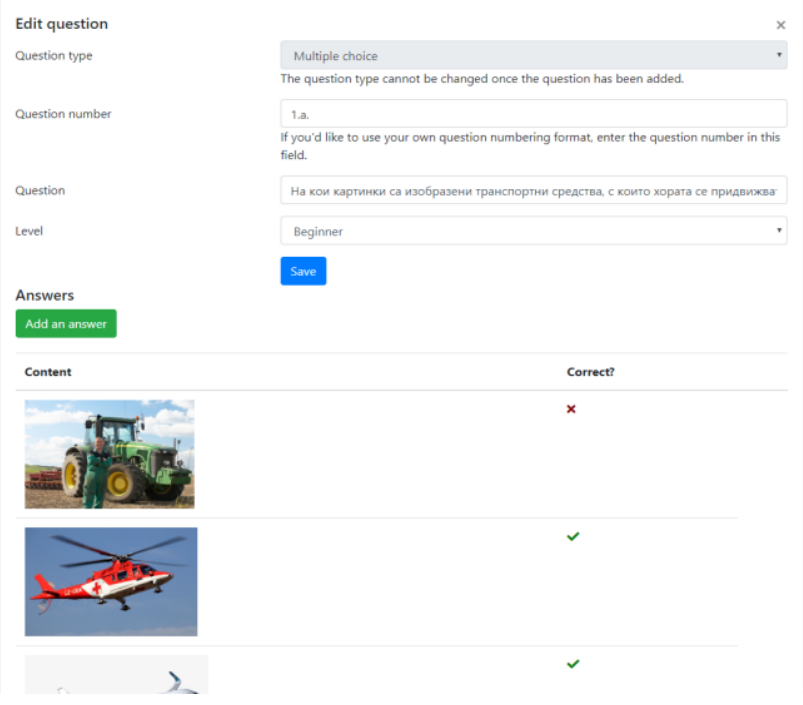

Figure 3. Question edit screen

On this screen, in addition to entering the question's text, teachers need to choose the question type, which has to be among the ones mentioned above. An option to enter a custom question number allows more flexibility and numbering question according to different criteria, including (but not limited to) level of difficulty (for example, 1.a instead of 1 for easy, 1.b instead of 2 for intermediate, and 1.c instead of 3 for advanced). Furthermore, the difficulty level can be indicated using the Level dropdown, which helps with data analysis in later stages of the screening process.

The last and crucial part of the question setup is entering the question's answer options and indicating the correct answer; the process differs slightly depending on the question type, but some common traits are shared by all question types:

- Teachers can use rich text formatting as well as embed multimedia content in the answers thanks to the rich text editor. It is shown in

- Figure 4. Advanced users familiar with web technologies may directly enter custom HTML code.

- An image manager is also included so that images and other external content can be uploaded to the server and hosted in a dedicated part of the filesystem. This reduces reliance on external storage systems and gives the system a certain degree of autonomy.

The correct answer can be selected by:

- Marking the correct answers using the checkmarks for single-choice and multiplechoice questions.

- Using drag-and-drop for connect and group types; these types allows teachers to create questions where students can connect related answers to each other.

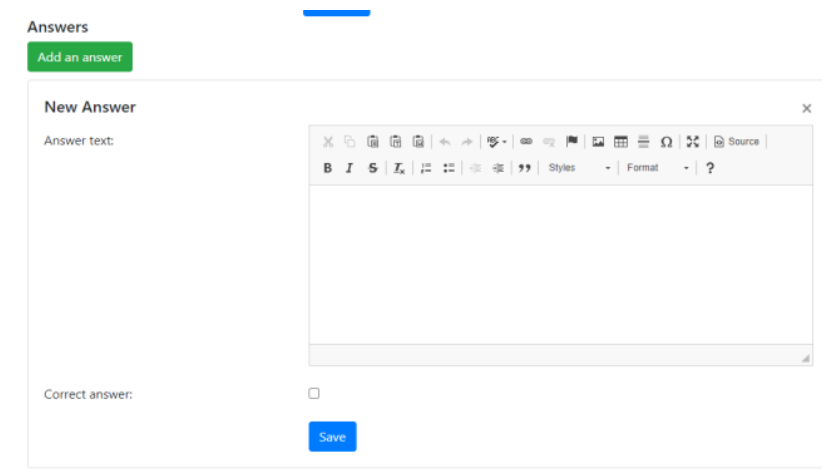

Figure 4. The rich text editor for answers

Tests created via the system can be printed out and handed to students if enough computing devices are not available. When students have filled out the paper tests, teachers can enter their results into the system for data storage and analysis. The screen used to input results into the system is shown in Figure 5.

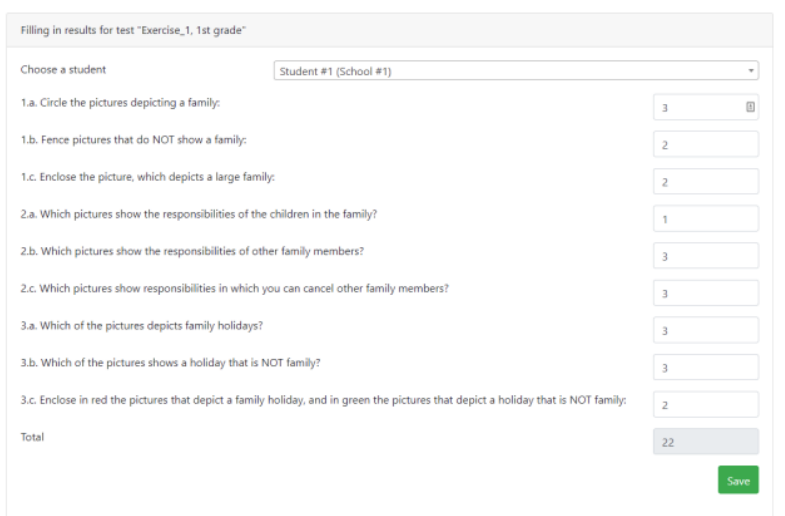

Figure 5. Screen used by teachers to enter test results

Data collected via test system can then be used with the statistics module, which allows certain data aggregation and analysis operations based on the current contents of the database. The main page of the statistics module is shown in Figure 6.

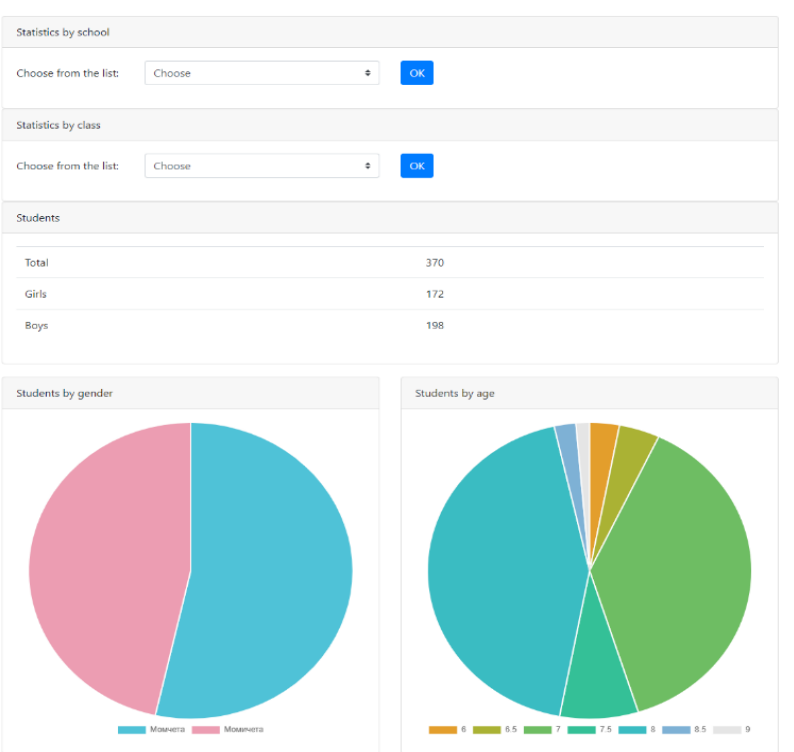

Figure 6. The statistics module's main page 
Using this module, teachers can get insights on their test results, and can filter data by school, class or age group. If the provided data analysis options are not complex enough for a particular requirement, the system allows exporting anonymized variants of the collected data in various machine-readable formats, such as CSV, Excel and JSON for further analysis with various professional statistics, business intelligence and data analysis tools.

When working with learners, it is inevitable that some personal information, such the learners' names may be collected for identification purposes. The security of that information needs to be taken extremely seriously. Special care has been taken so that the testing system supports the following security requirements:

- Non-requirement for personal information. The system can be used without entering data that can identify learners, such as their names. However, if such a need arises and the institution using the software is a personal data administrator, they can enter a limited subset of identifying information into the system.

- Even though names, email addresses and other personal information might be stored, it is completely anonymized for statistics and data analysis purposes. Each learner is assigned with an anonymized identifier which is used during data export and analysis, which serves to prevent personal information leaks from exported data.

- Personal information can be fully deleted at the learner's (or their legal guardian's) request.

- It is mandatory that the connection between the screening system and other personalized learning modules is fully and properly encrypted with the TLS protocol. Encryption is also required between the end user's device and the screening system.

The screening software's development leverages modern web technology to deliver a consistent experience regardless of the type of device used to access it. It has been developed using the Laravel 5 framework and PHP in combination with the MySQL database management system. The jQuery, Vue.js and Bootstrap frameworks are used for the frontend development, along with HTML and CSS, and JavaScript; AJAX requests are implemented for a number of features to reduce loading unnecessary elements and make the user experience smoother.

\section{Results}

To assess the effectiveness of the system, an empirical study was conducted. It was carried out within one school year. The main object of the empirical study relates to forming social skills through the model of 'Pedagogical technologies for inclusive education' developed and implemented in the lesson on the subject "Around the World".

The study was attended by 370 students aged between 6 and 9 years, in two local schools. The study of the input level of cognitive activity from the control and experimental group is realized in the first weeks of the school year. Through the created didactic tests, respectively for the first and second grade, testing of the students was conducted. Tests are a measuring tool the level of student achievement. They consist of a specific number of questions with progressively increasing levels of difficulty:

- 1.a (beginner level - showed poor results);

- 1.b (intermediate level - students showed satisfactory results);

- $\quad$ 1.c (advanced level - students with results giving grounds for outstanding gifts and talents).

To establish the degree of the set goal, at the end of the school year a survey was conducted on the initial level of students' cognitive activity.

Figure 7 shows the distribution of the 87 first graders from the control and 87 first graders from the experimental group by levels.

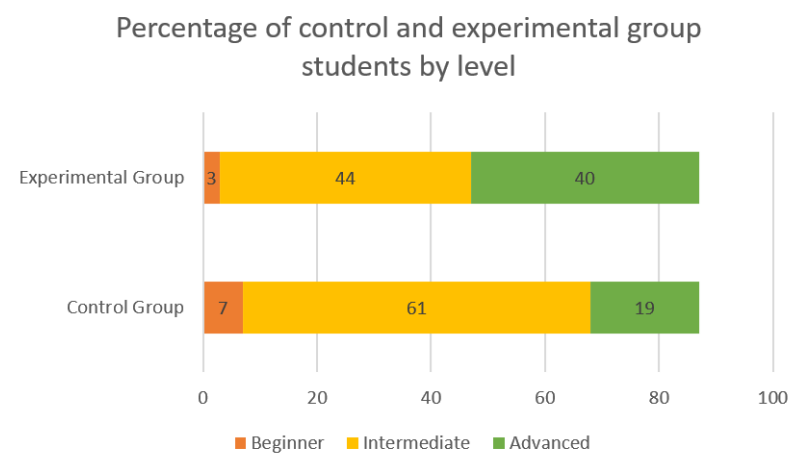

Figure 7. Distribution of control and experimental group students by level in the first grade

7 students from control group or $8 \%$ fall into the beginner level of the test. At the experimental group 3 students fall to a low level or 3\%. The majority of the surveyed first graders fall into the intermediate level of control group, namely 61 or $70 \%$, while 44 of the students or $51 \%$ are in experimental group. 19 or $22 \%$ of the trainees belong to an advanced level of control group. 40 of the surveyed pupils or $46 \%$ reach an advanced level in experimental group.

The comparative analysis of the presented results shows that 5\% less students from experimental group fall into a beginner level of the test, compared to control group. On intermediate level, control group has $19 \%$ more students than experimental group. Advanced level reach $24 \%$ more surveyed first graders than experimental group, compared to control group. 
High positive results of both groups are a clear indicator that entering first grade, they have a solid knowledge of their social environment.

Figure 8 shows the distribution of 98 students from control group and 98 from experimental group who completed the Incoming diagnostics test for the second grade.

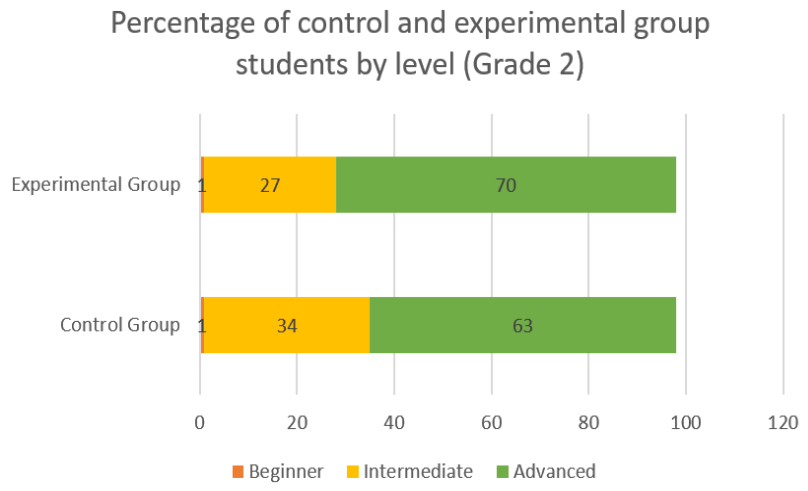

Figure 8. Distribution of control and experimental group students by level in the second grade

Only 1 student or $1 \%$ of both studied groups fall into the beginner level of the test. In the intermediate level of control group 34 or $35 \%$, while in experimental group are 27 of the students or $28 \% .63$ of the trainees or $64 \%$ of control group belong to an advanced level. 70 of the second graders or $71 \%$ of experimental group reach an advanced level.

The positive results, achieved by both groups, are an indicator that during their education in the first grade they have gained lasting knowledge about the social environment around them, with which they enter the second grade.

Figure 9 shows the distribution of the 87 students from control and 87 from experimental group who filled in the Outgoing Diagnostics test for first grade.

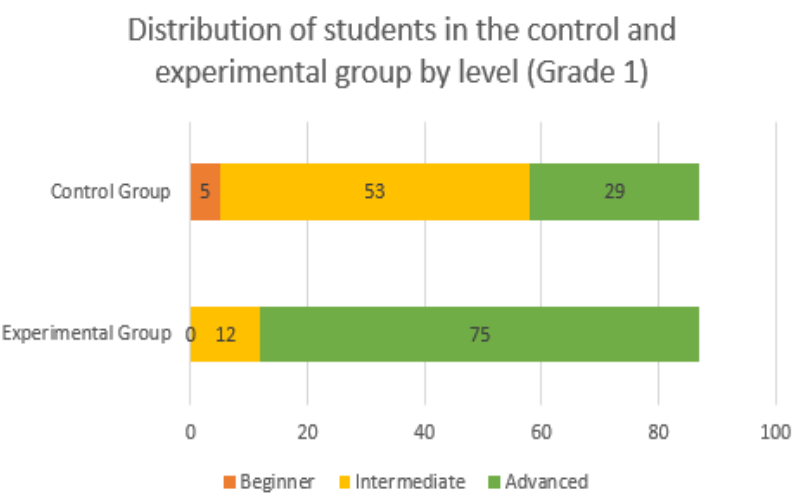

Figure 9. Distribution of control and experimental group students by level in the first grade

The beginner level of the test includes 5 students or $6 \%$ of the control group and none of the experimental. At the intermediate level 53 students or $61 \%$ of the control group, while at the experimental group are 12 of the students or 14\%. 29 of the trainees from control group or $33 \%$ belong to an advanced level. In experimental group, 75 of the surveyed students or $86 \%$ reach advanced level, achieving a 53\% higher result compared to control group.

The percentage distribution of the achieved results of the 194 pupils from control and experimental group in relation to the maximum number of test points was shown in Figure 10.

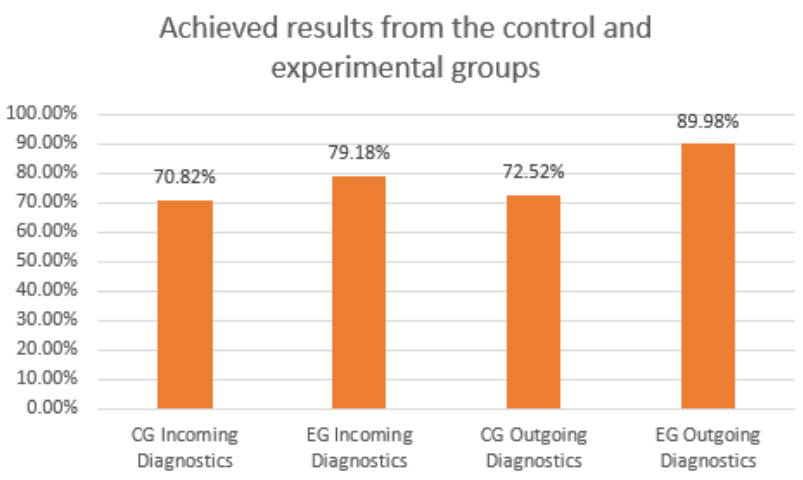

Figure 10. Percentage distribution of the achieved results from control and experimental group

The comparative analysis of the results of the input and output diagnostics tests presented by the two groups shows the following: students from the control group demonstrate $70.82 \%$ on the input diagnostics test and experimental group with $8.36 \%$ higher achievement, or $79.18 \%$ in the experimental group. In the outgoing diagnostic test, the results are as follows: $72.52 \%$ for control group and $89.98 \%$ for experimental group, achieving an improvement of $17.46 \%$ over control group.

The percentage distribution by levels of the 196 pupils from the control and experimental group who participated in the Outgoing Diagnosis test for second grade is shown in Figure 11.

\section{Distribution of students in the control and experimental group by level (Grade 2)}

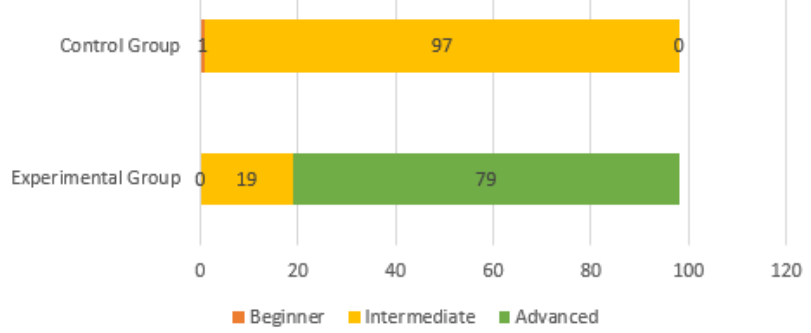

Figure 11. Distribution of control and experimental group students by level in the second grade

$1 \%$ of the students from the control and none of the students from the experimental group fall into the beginner level of the test. At the intermediate level 
97 or $99 \%$ of the students from control group, while in experimental group are 19 of the students or $19 \%$. $0 \%$ refer to an advanced level of control group. In experimental group 79 of the surveyed second graders or $81 \%$ reach an advanced level, achieving an $81 \%$ higher result compared to control group.

The percentage distribution of the achieved results of the 196 pupils from control and experimental group in relation to the maximum number of points on the test is shown in Figure 12.

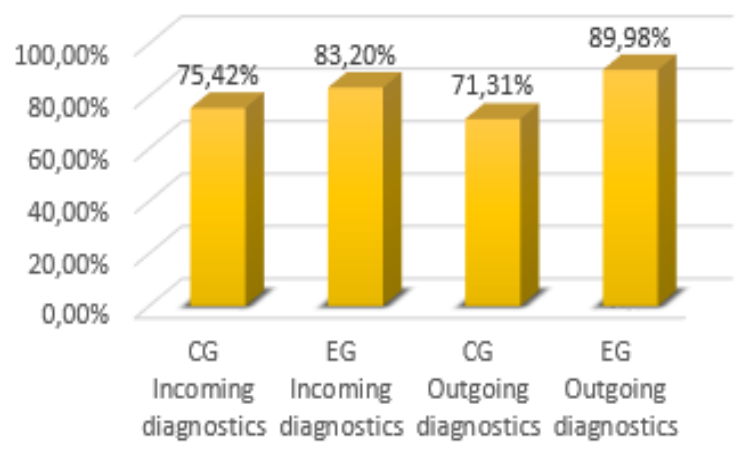

Figure 12. Percentage distribution of the achieved results from control and experimental group

The presented results of the two groups participating in the Incoming and Outgoing diagnostics tests are as follows: the students from control group demonstrate $75.42 \%$ on the test for Incoming diagnostics, and experimental group with $7.78 \%$ higher achievement or $83.20 \%$. The results on the Outgoing Diagnosis test, are as follows: $71.31 \%$ for control group and $90.52 \%$ for experimental group, making a progress of $19.21 \%$ compared to control group.

\section{Conclusion}

Personalized learning systems and approaches have been found to improve engagement among learners and generally lead to better performance in subsequent assessments.

Using the data obtained during the study and analyzed with the help of the screening system, it was found that the progress of the students from the experimental group compared to those from the control group on the test for outgoing diagnosis for first grade is $17.46 \%$, and for students from second grade $-19.21 \%$, proving the effectiveness of the integrated personalized learning system.

The development of the full personal potential of each child is possible by updating and modernizing the ways of teaching, through the inclusion of methods, tools, technologies, etc., to create a supportive and tolerant environment for students to be able to participate, adapt and socialize in the life of modern society.

\section{Acknowledgements}

The study was supported by contract of University of Ruse “Angel Kanchev", № FNI-RU-01, ,, Design and Construction of a Smart Teaching and Research Laboratory for Doctoral Students - Part II".

\section{References}

[1]. Ng, P. T. (2021). Timely change and timeless constants: COVID-19 and educational change in Singapore. Educational Research for Policy and Practice, 20(1), 19-27. https://doi.org/10.1007/s10671-020-09285-3

[2]. Ivanova, G., Zlatarov, A. P. P., Baeva, P. A. D., \& Antonova, D., (2020). New Approaches In Doctoral Education at The University of Ruse - A Response To The Challenges of A New Era. Proceedings Of University of Ruse, volume 59, book 9.1.

[3]. Kunev, S., Fleaca, B., Antonova, D., \& Dráb, R. (2020, November). Fostering the innovative university student-centred learning by application of ICT tools together with stakeholders: a project methodology overview. In 2020 7th International Conference on Energy Efficiency and Agricultural Engineering (EE\&AE) (pp. 1-4). IEEE.

[4]. Loukantchevsky, M., Kostadinov, N., \& Avakyan, H. (2019, June). A Testbed of Non-determinism in Educational Context. In Proceedings of the 20th International Conference on Computer Systems and Technologies (pp. 304-307).

[5]. Chen, C. M. (2008). Intelligent web-based learning system with personalized learning path guidance. Computers \& Education, 51(2), 787-814.

[6]. Berghel, H. (1997). Cyberspace 2000: Dealing with information overload. Communications of the ACM, 40(2), 19-24.

[7]. Ivanova, E., \& Doncheva, J. (2018). The efficiency in training and knowledge development to students by integrating the information and communication technologies in the lesson of â€ $€^{\sim}$ Around the worldâ€ ${ }^{\mathrm{TM}}$. New Trends and Issues Proceedings on Humanities and Social Sciences, 5(5), 85-90.

[8]. Tsolis, D., Stamou, S., Christia, P., Kampana, S., Rapakoulia, T., Skouta, M., \& Tsakalidis, A. (2010). An adaptive and personalized open source e-learning platform. Procedia-Social and Behavioral Sciences, 9, 38-43.

[9]. Hübscher, R. (2000, August). Logically optimal curriculum sequences for adaptive hypermedia systems. In International conference on adaptive hypermedia and adaptive web-based systems (pp. 121-132). Springer, Berlin, Heidelberg.

[10]. Ivanova, E., \& Doncheva, J., (2019). Pedagogical Technologies For Inclusive Education Of Pupils In The Initial Stage Of Education On The Subject 'Around The World'. Proceedings of INTED2019 Conference 11th-13th March 2019, Valencia, Spain.

[11]. Manukova, A., Marinov, M., \& Grozeva, M. (2019). An Approach to Evaluation of Clinically Healthy People by Preventive Cardio Control. Cybernetics and Information Technologies, 19(2), 133-145. https://doi.org/10.2478/cait-2019-0020 
TEM Journal. Volume 10, Issue 3, Pages 1454-1460, ISSN 2217-8309, DOI: 10.18421/TEM103-58, August 2021.

[12]. Zlatarov, P., Ivanova, G., \& Baeva, D. (2019, May). A web-based system for personalized learning path tracking of doctoral students. In 2019 42nd International Convention on Information and Communication Technology, Electronics and Microelectronics (MIPRO) (pp. 773-778). IEEE.
[13]. Fischer-Hübner, S., Martucci, L. A., Fritsch, L., Pulls, T., Herold, S., Iwaya, L. H., ... \& Zuccato, A. (2018, September). A MOOC on privacy by design and the GDPR. In IFIP World Conference on Information Security Education (pp. 95-107). Springer, Cham. 